\title{
The Crystal Structure of Sulvanite, $\mathrm{Cu}_{3} V S_{4}$.
}

\author{
By \\ Linus Pauling and Ralph Hultgren in Pasadena.
}

(With 3 figures.)

Massive sulvanite, $C u_{3} V S_{4}$, from Burra Burra in Australia was recognized as cubic by $\mathrm{Orcel}^{1}$ ) and de $\mathrm{Jong}^{2}$ ) from its isotropic reflection of polarized light. De Jong prepared powder X-ray photographs of the substance, and assigned indices to the lines on the basis of a cubic unit with $a_{0}=10.75 \AA$, containing $8 \mathrm{Cu}_{3} V S_{4}$. Assuming chemically identical atoms to be crystallographically equivalent, he suggested a complicated arrangement of the atoms within this unit.

We noticed that the experimental data published by de Jong do not require such a large unit, but can be accounted for by a unit with $a_{0}=5.375 \AA$, containing $1 C u_{3} V S_{4}$, and we planned to study the crystal further. The opportunity to do this arose when a new find of sulvanite, from near Mercur, Utah, was made ${ }^{3}$ ), consisting of cleavable masses and a few individual crystals. Dr. W. T. Schaller of the United States Geological Survey very kindly lent us this material, enabling us to prepare excellent Laue and oscillation photographs. Data from these show conclusively that the true unit contains only one molecule. A rigorous derivation has led to a very interesting new type of atomic arrangement.

\section{The unit of structure and space-group symmetry.}

Several oscillation photographs were prepared of the radiation from a molybdenum-target tube, either unfiltered or filtered through zirconia, reflected from (100) of a crystal of sulvanite oscillating through $45^{\circ}$ about a vertical [010] axis. A cleavage face about $2.5 \mathrm{~mm}$. across and a smaller wellformed cube about $0.8 \mathrm{~mm}$. on edge were used. Various orders of (100) up to (800) were observed to reflect, with the estimated relative intensities given in table $\mathrm{I}$.

1) J. Orcel, Bl. Soc. franç. Min. 51, 205. 1928.

2) W. F. de Jong, Z. Krist. 68, 522. 1928.

3) C. A. Schempp and W. T. Schaller, Am. Min. 16, 557. 1931. 
Table I.

Oscillation data from (100) of sulvanite.

\begin{tabular}{c|c|c|c|c|c|c}
\hline X-ray Line & $\begin{array}{c}\text { Wave } \\
\text { Length }\end{array}$ & Order & $\begin{array}{c}\text { Angle of } \\
\text { Reflection }\end{array}$ & $d_{100}$ & $\begin{array}{c}\text { Estimated } \\
\text { Intensity }\end{array}$ & $\begin{array}{c}\text { Calculated } \\
\text { Intensity for } \\
u=0.235\end{array}$ \\
\hline \hline MoK $\beta$ & 0.6311 & 1 & $3^{\circ} 23^{\prime}$ & 5.348 & & \\
$\alpha$ & .7093 & 1 & $3^{\circ} 47^{\prime}$ & 5.365 & 5 & 37 \\
$\gamma$ & .6197 & 2 & $6^{\circ} 38^{\prime}$ & 5.361 & & \\
$\beta$ & & 2 & $6^{\circ} 45^{\prime}$ & 5.371 & & \\
$\alpha_{1}$ & .70783 & 2 & $7^{\circ} 35^{\prime}$ & 5.366 & 3 & 19 \\
$\alpha_{2}$ & .71212 & 2 & $7^{\circ} 37^{\prime}$ & 5.375 & & \\
$\beta$ & & 3 & $10^{\circ} 10^{\prime}$ & 5.367 & & \\
$\alpha_{1}$ & & 3 & $11^{\circ} 24^{\prime}$ & 5.371 & 0.8 & 4.0 \\
$\alpha_{2}$ & & 3 & $11^{\circ} 28^{\prime}$ & 5.370 & & \\
$\gamma$ & & 4 & $13^{\circ} 20^{\prime}$ & 5.372 & & \\
$\beta$ & & 4 & $13^{\circ} 36^{\prime}$ & 5.371 & & \\
$\alpha_{1}$ & & 4 & $15^{\circ} 17^{\prime}$ & 5.371 & 10 & 53 \\
$\alpha_{2}$ & & 4 & $15^{\circ} 23^{\prime}$ & 5.368 & & \\
$\alpha_{1}$ & & 5 & $19^{\circ} 14^{\prime}$ & 5.374 & & \\
$\alpha_{2}$ & & 5 & $19^{\circ} 22^{\prime}$ & 5.370 & 1 & 7.4 \\
& & 6 & & & Absent & 1.6 \\
& & 7 & & & Absent & 0.5 \\
& & 8 & & & 1 & 6.6
\end{tabular}

Weighted mean : $d_{100}=\mathbf{5 . 3 7 0} \AA$.

The value of $a_{0}=5.370 \pm 0.005 \AA$ (table I) was obtained from a photograph of radiation reflected on the same plate from (100) of sulvanite and (0001) of $\beta$-alumina, for which a spacing of $11.225 \AA$ was assumed $\left.^{1}\right)$. This agrees well with the value $\frac{1}{2} \times 10.750=5.375 \AA$ from de Jong.

Laue photographs were prepared in the usual way from two small crystals (described by Schempp and Schaller), the incident beam being normal to (100), (110), or (111), or making small angles with the normals to these faces. On assigning indices to the reflections with the aid of gnomonic projections and calculating values of $n \lambda$ on the basis of a unit with $a_{0}=5.37 \AA$, it was found that none of the values was smaller than $0.24 \AA$, the short wave-length limit of the incident radiation. Consequently there is no evidence requiring a larger unit of structure; and in view of the excellence of the Laue photographs obtained and the

1) Linus Pauling and A. Björkeson, Pr. Nat. Acad. Am. 11, 445. 1925. 
sensitivity of the Laue method of determining the true unit ${ }^{1}$ ), the unit with

$$
\underline{a_{0}=5.370 \AA}
$$

may be confidently accepted as the true one. With $1 \mathrm{Cu}_{3} V S_{4}$ in the unit, the calculated density is $3.94 \mathrm{~g} . / \mathrm{cm}^{3}$, in satisfactory agreement with the reported values $\left.{ }^{2}\right)^{3}$ ) $3.98,4.01$, and 4.03 .

Many observed reflections eliminate the body-centered and facecentered lattices, requiring the lattice to be simple cubic. Lave photographs through (100), (110), and (111) show the symmetry elements expected for $O, T_{d}$, and $O_{h}$, rather than $T$ and $T_{h}$. (It may be mentioned that photographs showing a fourfold and a three-fold axis were obtained from the same crystal, which is thus proved to be cubic.) The possible space groups are thus limited to $T_{d}^{1}, T_{d}^{4}, O^{1}, O^{2}, O^{6}, O^{7}, O_{h}^{1}, O_{h}^{2}, O_{h}^{3}$, and $O_{h}^{4}$. The observed reflections (100), (300), and many others eliminate $T_{d}^{4}, O^{2}$, $O^{6}, O^{7}, O_{h}^{2}, O_{h}^{3}$, and $O_{h}^{4}$, leaving only $T_{d}^{1}, O^{1}$, and $O_{h}^{1}$. These three space groups are indeed the only ones which provide positions for the atoms $V$, $3 C u, 4 S$ in a cubic unit.

The space groups $O^{1}$ and $O_{h}^{1}$ provide the possible positions

1a. 000

1b. $\quad \frac{1}{2} \frac{1}{2}$

3a. $0 \frac{1}{2} \frac{1}{2}, \frac{1}{2} 0 \frac{1}{2}, \frac{1}{2} \frac{1}{2} 0$

3 b. $\quad \frac{1}{2} 00, \quad 0 \frac{1}{2} 0, \quad 00 \frac{1}{2}$

for the atoms in the unit. For any atomic arrangement based on these positions the structure factor becomes $F=f_{V}+3 f_{C u}+4 f_{S}$ for all even order reflections, which should consequently show a normal decline in intensity with decreasing interplanar distance. The observation that (400) reflects more strongly than (200) consequently eliminates all such arrangements. The space group is hence determined to be

$$
\underline{T_{d}^{1}-P \overline{4} 3 m} .
$$

\section{The atomic arrangement.}

The possible atomic positions provided by $T_{d}^{1}$ are $1 \mathrm{a}, 1 \mathrm{~b}, 3 \mathrm{a}, 3 \mathrm{~b}$, and, in addition,

4a. $u u u, \quad u \bar{u} \bar{u}, \quad \bar{u} u \bar{u}, \quad \bar{u} \bar{u} u$.

1) Illustrated, for example, by the work of R. G. Dickinson, J. Am. chem. Soc. 45, 958. 1923; Z. Krist. 64, 400. 1926, on tin tetraiodide, and of Linus Pauling and L. O. Brockway, Z. Krist. 82, 188. 1932, on chalcopyrite.

2) G. A. Goyder, J. chem. Soc. London 77, 1094. 1900.

3) H. Schultze and H. Steinmetz, Z. Krist. 49, 40. 1911. 
The sulfur atoms are shown by the fore-going argument to occupy $4 \mathrm{a}$. The vanadium atom may be placed in 1 a rather than $1 \mathrm{~b}$ without loss of generality. Two distinct structures are then obtained by placing $3 \mathrm{Cu}$ in $3 \mathrm{a}$ or in $3 \mathrm{~b}$. These are the only possible atomic arrangements.

It is easily shown that the observed intensities of reflection in various orders from (100) require that for the first of these two arrangements the parameter lie in the neighborhood of $\frac{1}{4}$; at $u=0.27 \pm 0.02$ the best agreement is obtained, though this is not very good, the observed rather strong first-order reflection not being well accounted for. This arrangement is a very simple and reasonable one, obtained from the sphalerite arrangement by replacing one of the zinc atoms by vanadium and the other three by copper. The approximation of the value of $a_{0}$ to that for sphalerite, $5.42 \AA$, further suggests this structure. Chalcopyrite, $C u F e S_{2}$, has been shown ${ }^{1}$ ) to have a structure similarly related to that of sphalerite. Despite its reasonableness, however, this is not the structure of sulvanite, for many intensity comparisons on Laue photographs are incompatible with this atomic arrangement. For example, (643) is observed to reflect on Laue photographs about as intensely as (553), whereas the calculated intensity of reflection of (553) is over ten times that of (643) throughout the parameter range $0.20<u<0.30$. It is, indeed, evident from the nature of the photographs that the structure of sulvanite is not closely related to the sphalerite structure; for inasmuch as the $f$-values for $V$ and $C u$ are not very different, reflections other than those with $h, k, l$ either all odd or all even should be very weak (as was the case in chalcopyrite, where such reflections were not observed at all in the first two investigations of this crystal and appeared only as very weak Laue spots in the last study ${ }^{1}$ ), whereas actually on the sulvanite Laue photographs the strongest reflections are those from planes not satisfying this condition.

The parameter for the remaining structure, with $3 \mathrm{Cu}$ in $3 \mathrm{~b}$, can be limited to a narrow range of values with the aid of intensity comparisons on oscillation and Laue photographs. In Fig. 1 there are plotted the intensities of reflection in various orders from (100) for the parameter range 0.20 to 0.30 (limitation to this region being easily made) calculated from the equation

$$
\begin{gathered}
I_{n 00}=\text { Constant } \cdot n \cdot \frac{1+\cos ^{2} 2 \theta}{2 \sin 2 \theta} \\
\cdot\left\{f_{0_{V}}+f_{0_{C u}}\left(2+\not / \cdot(-1)^{n}\right)+4 f_{0_{S}} \cos 2 \pi n u\right\}^{2} \cdot e^{-0.125 d_{n}^{2}{ }_{n 00}} .
\end{gathered}
$$

1) Linus Pauling and L. O. Brockway, Z. Krist. 82, 188. 1932. 
The factor $n$ is required by the experimental conditions, under which the amount of incident radiation intercepted by the face of the crystal increases linearly with the order of reflection. The temperature factor corresponds to an estimated characteristic temperature of about $530^{\circ}$ The $f_{0}$-values used are those of $\mathrm{Pauling}$ and Sherman $\left.{ }^{1}\right)$. It is seen that the observed intensity relations $(800) \gg(600)$ and $(500)>(300)$ limit $u$ to between 0.223 and 0.239 , the quantitative agree-

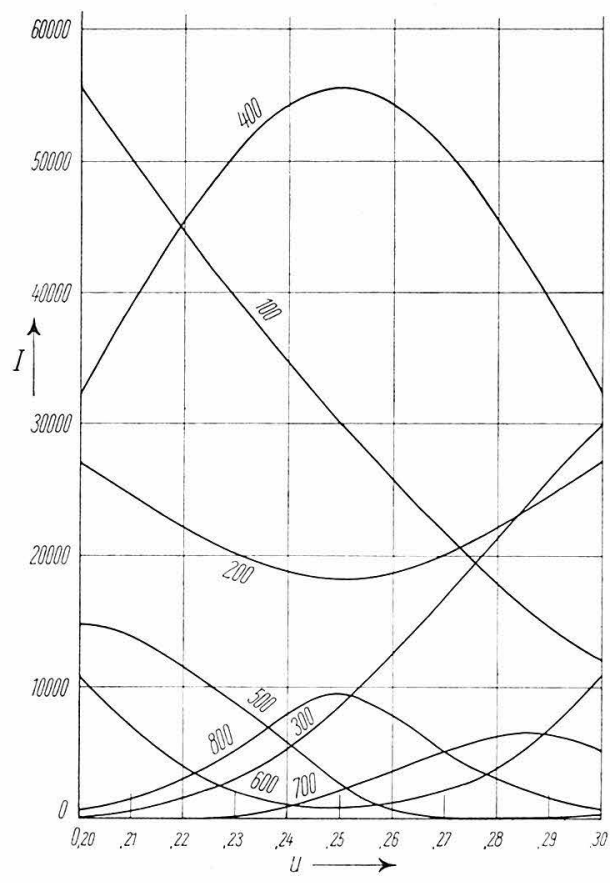

Fig. 1.

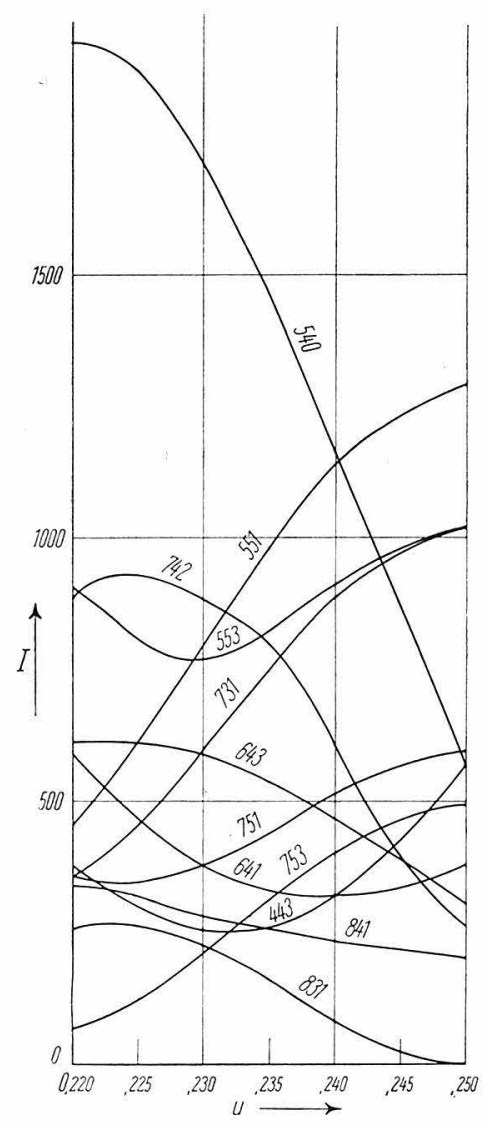

Fig. 2.

Fig. 1. Calculated intensities for various orders of reflection from (100) of sulvanite as functions of $u$.

Fig. 2. Calculated intensities of Laue reflections.

ment being most satisfactory in the neighborhood of 0.235 . The intensity relation $(731)>(643)$ on Laue photographs further requires that $u$ be greater than 0.230 , as is seen from Figure 2 , in which there are plotted some intensity curves calculated for a wave-length of $0.40 \AA$ with the expression

1) Linus Pauling and J. Sherman, Z. Krist. 81, 1. 1932. 


$$
I_{h k l}=\text { Constant } \cdot \frac{1+\cos ^{2} 2 \theta}{2 \sin 2 \theta}\left|F_{0 h k l}\right|^{2} e^{-0.125 / d^{2} h k l}
$$

with

$$
F_{0}=\sum_{i} f_{0 j} e^{2 \pi i\left(h x_{j}+k y_{j}+l z_{j}\right)} .
$$

(The use of Lorentz and polarization factors not corresponding exactly to the Laue technique probably introduces no appreciable error.) The parameter accordingly has the value

$$
u=0.235 \pm 0.004 \text {. }
$$

The agreement between observed and calculated intensities is shown in Tables I and II.

Table II.

Data from Laue photographs 1 and 6 .

\begin{tabular}{|c|c|c|c|c|c|c|}
\hline \multirow{2}{*}{$\begin{array}{l}h k l \\
211\end{array}$} & \multirow{3}{*}{$\begin{array}{l}d_{h k l} \\
2.19 \AA \\
1.79\end{array}$} & \multirow{2}{*}{$\begin{array}{c}\text { Calculated } \\
\text { Intensity for } \\
u=0.235 \\
6\end{array}$} & \multicolumn{2}{|c|}{$\begin{array}{l}\text { Observed Intensity } \\
\text { La u e } 1\end{array}$} & \multicolumn{2}{|c|}{$\begin{array}{l}\text { Observed Intensity } \\
\text { Laue } 6\end{array}$} \\
\hline & & & 2.5 & $0.44 \AA$ & & \\
\hline 221 & & 63 & 25 & .40 & & \\
\hline 311 & 1.62 & 126 & 15 & .28 & & \\
\hline 320 & 1.53 & 60 & & & 25 at & $0.39 \AA$ \\
\hline 321 & 1.44 & 2 & 0.4 & .41 & & \\
\hline 322 & 1.30 & 11 & 1.0 & .30 & 12 & .38 \\
\hline 331 & 1.23 & 60 & 5 & .30 & & \\
\hline 421 & 1.17 & 13 & 5 & .42 & 13 & .39 \\
\hline 332 & 1.15 & 2 & & & 0.5 & .39 \\
\hline 430 & 1.07 & 5 & & & 3.5 & .37 \\
\hline 431 & 1.05 & 3 & 0.4 & .36 & 0.5 & .35 \\
\hline 510 & 1.05 & 0 & 0.0 & .32 & & \\
\hline 432 & 1.00 & 18 & 2.5 & .35 & & \\
\hline 520 & 1.00 & 2 & 0.3 & .38 & & \\
\hline 441 & 0.94 & 10 & 1.5 & .44 & & \\
\hline 433 & .92 & 2 & 0.1 & .39 & & \\
\hline 531 & .91 & 23 & 3.5 & .37 & 10 & .38 \\
\hline 611 & .87 & 4 & & & 0.7 & .40 \\
\hline 540 & .84 & 15 & 1.4 & .40 & & \\
\hline 443 & .84 & 3 & 0.4 & .39 & & \\
\hline 533 & .82 & 14 & & & 7 & .40 \\
\hline 542 & .80 & 1 & & & 0.3 & .37 \\
\hline 632 & .77 & 2 & 0.4 & .42 & 0.5 & .42 \\
\hline 551 & .75 & 10 & 1.0 & .43 & & \\
\hline 711 & .75 & 10 & 0.6 & .35 & & \\
\hline
\end{tabular}

Laue 1. Incident beam $8^{\circ}$ from [110]

Laue 6. Incident beam $2^{\circ}$ from $[110]$ 
Table II (continuation).

\begin{tabular}{c|c|c|cc|cc}
\hline hkl & $d_{h k l}$ & $\begin{array}{c}\text { Calculated } \\
\text { Intensity for } \\
u=0.235\end{array}$ & \multicolumn{2}{|c|}{$\begin{array}{c}\text { Observed Intensity } \\
\text { Laue 1 }\end{array}$} & \multicolumn{2}{|c}{$\begin{array}{c}\text { Observed Intensity } \\
\text { Laue }\end{array}$} \\
\hline \hline 641 & .74 & 3 & 0.3 & .38 & 0.7 & .41 \\
720 & .74 & 13 & 0.7 & .37 & & \\
553 & .70 & 8 & 0.6 & .37 & & \\
643 & .69 & 5 & 0.4 & .39 & 1.0 & .41 \\
652 & .68 & 7 & 0.3 & .37 & 0.7 & .37 \\
733 & .66 & 6 & & & 0.5 & .42 \\
742 & .65 & 8 & 0.4 & .39 & 0.6 & .37 \\
821 & .65 & 2 & 0.1 & .36 & & \\
830 & .63 & 1 & 0.05 & .39 & & .40 \\
831 & .63 & 2 & & & 0.2 & .42 \\
751 & .62 & 4 & & & 0.4 & .41 \\
841 & .60 & 2 & & & 0.3 & .43 \\
753 & .59 & 3 & & & 0.3 & .39
\end{tabular}

\section{Description of the structure.}

The atomic arrangement found for sulvanite is a new type, shown in Figure 3. Each copper atom is surrounded by four sulfur atoms at the corners of a nearly regular tetrahedron. Each vanadium atom is surrounded by four sulfur atoms at the corners of a regular tetrahedron. Each sulfur atom is surrounded by three copper atoms at three of the corners of a nearly regular tetrahedron, and a vanadium atom not at the fourth corner of the tetrahedron, but in the negative position to this; that is, in the pocket formed by the three copper atoms.

It is probable that there are bonds of essentially covalent type between each atom and its four nearest neighbors. The mutual orientation of the four sulfur bonds is very surprising; for in all other sulfur-containing covalent crystals whose structures are known with certainty, such as $\mathrm{ZnS}, \mathrm{MoS}_{2}, \mathrm{FeS} \mathrm{S}_{2}$, etc., the sulfur bonds are at approximately tetrahedral angles. It is true, however, that other angles may be expected on theoretical grounds, inasmuch as the $3 \mathrm{~d}$ as well as $3 \mathrm{~s}$ and $3 \mathrm{p}$ orbital wave functions may take part in bond formation ${ }^{1}$ ), in which case bonds at $110^{\circ}$ and $71^{\circ}$ may well be formed. The factors determining the stability of such bonds rather than tetrahedral bonds are not known. The following argument suggests that electrostatic interactions may be important in stabilizing the sulvanite structure. If each of the atoms in sulvanite forms four extreme covalent bonds, each consisting of two electrons

1) See R. Hultgren, Physic. Rev. 40, 891. 1932. 
shared equally between two nuclei, then the average electric charges on the various atoms will correspond to the formula $C u \equiv V_{3}^{+} S_{4}^{++}$. In the sulvanite structure the ions immediately adjacent to each ion are the same as in the sphalerite-type structure discussed above and eliminated by the X-ray data. In the next sphere of influence, however, there is a difference; in the sulvanite structure six of the negatively charged copper

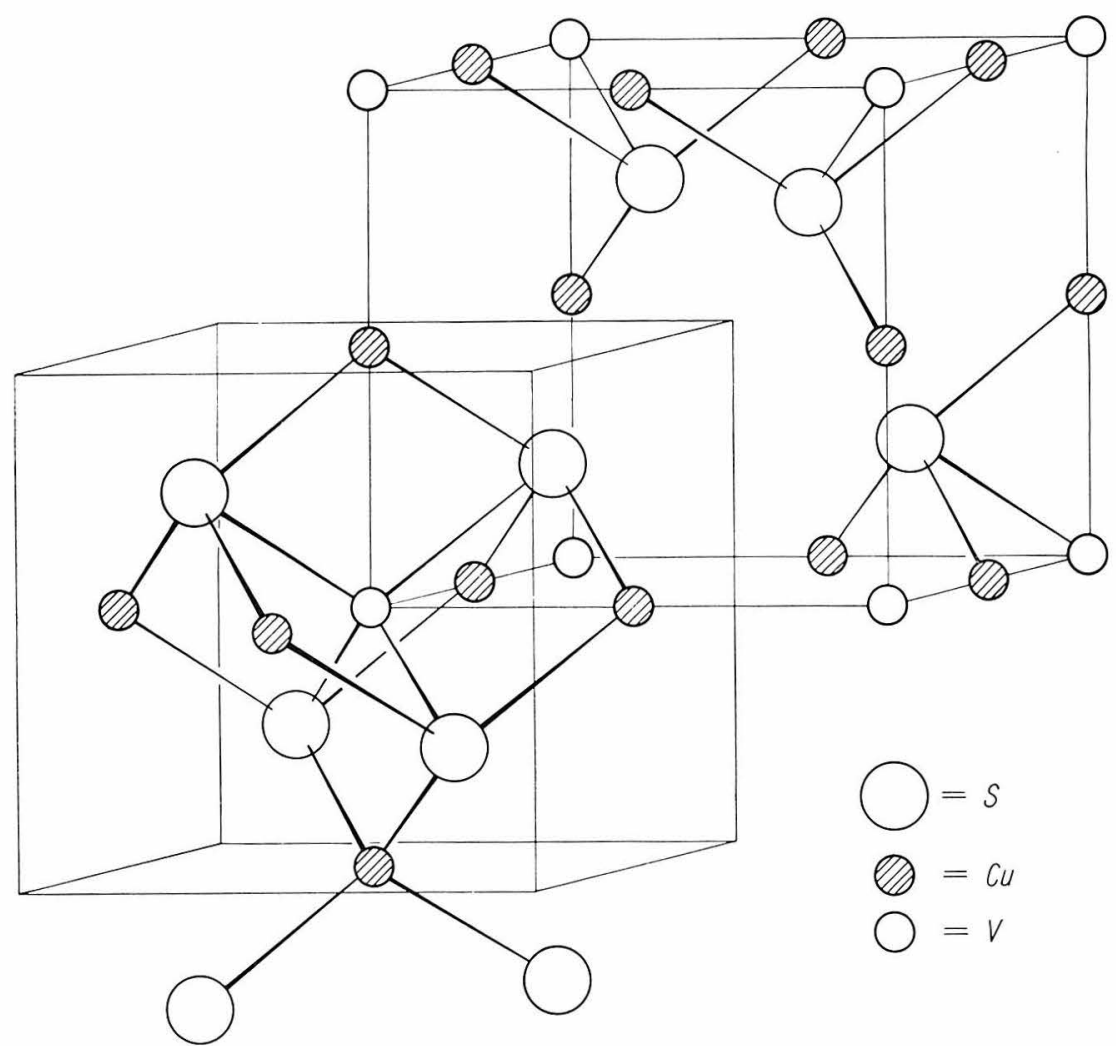

Fig. 3. The atomic arrangement in sulvanite, $C u_{3} V S_{4}$.

atoms are brought to within $\frac{1}{2} a_{0}$ of each positively charged vanadium atom, with a resultant stabilization of the structure because of the Coulomb terms. This is shown by the Madelung constants, which have the values 39.217174 for $\mathrm{Cu} \equiv \mathrm{V}^{+} \mathrm{S}_{4}^{++}$with the sulvanite structure and 37.026993 for the sphalerite-type structure (each for $u=\frac{1}{4}$, and referred to $a_{0}$ ), corresponding to an energy difference of $135 \mathrm{kcal} / \mathrm{mole}$ in favor of the sulvanite structure. This consideration is, of course, too idealized, for the bonds without doubt have considerable ionic character, which 
operates in such a direction as to make the metal atoms more positive and the sulfur atoms more negative ${ }^{1}$ ). If this occurs to the extent indicated by the formula $C u_{3}^{=} V^{++} S_{4}^{+}$, the Madelung constants become 17.249703 and 14.329462, respectively, the sulvanite structure being then favored by the energy difference $180 \mathrm{kcal} / \mathrm{mole}$. Only if the ionic character were to become so pronounced as to make the copper atoms positive would the Madelung constant for the sulvanite structure fall below that for the sphalerite-type structure.

The interatomic distances found are $V-S=2.186 \pm 0.040 \AA$ and $C u-S=2.285 \pm 0.014 \AA$. The $C u-S$ distance is somewhat smaller than the sum of the tetrahedral radii $^{2}$ ) for sulfur and univalent copper, $2.39 \AA$. As in the case of chalcopyrite, this probably indicates that the valence states are not well defined as $C u_{3}^{I} V^{V} S_{4}$, but fluctuate, the copper resonating between cuprous and cupric states and the vanadium between quinquivalent and lower states.

\section{Summary.}

The study by means of Laue and oscillation photographs of the cubic mineral sulvanite, using single crystals from Mercur, Utah, has shown the unit of structure to have $a_{0}=5.370 \pm 0.005 \AA$, and to contain $1 \mathrm{Cu}_{3} V S_{4}$. The space group is $T_{d}^{1}-P \overline{4} 3 \mathrm{~m}$. The atomic arrangement is a new type, $V$ at $000,3 C u$ at $\frac{1}{2} 00,0 \frac{1}{2} 0,00 \frac{1}{2}$, and $4 S$ at $u u u, u \bar{u} \bar{u}, \bar{u} u \bar{u}$, $\bar{u} \bar{u} u$, with $u=0.235 \pm 0.004$. Smallest interatomic distances are $V-S=2.186 \pm 0.040 \AA$, and $C u-S=2.285 \pm 0.014 \AA$.

We are indebted to Dr. W. T. Schaller of Washington, D. C., for lending us the excellent crystals of sulvanite for investigation, and to Dr. J. Sherman of this Laboratory for the calculation of the Madelung constants.

July 22nd, 1932. Contribution No. 332 from the Gates Chemical Laboratory, California Institute of Technology.

Received August 7th, 1932.

1) For a discussion of ionic character of covalent bonds see Linus Pauling, Pr. Nat. Acad. Am. 18, 414. 1932; J. Am. chem. Soc. 54, 3570. 1932.

2) Linus Pauling and M. L. Huggins, Z. Krist. to be published. 